\title{
FLASH
}

\section{UNE TRANSLOCATION CHROMOSOMIQUE PERMET DE CARACTÉRISER LE GĖNE DE LA POLYKYSTOSE RÉNALE AUTOSOMIQUE DOMINANTE-1}

Quelques mois seulement après l'identification et la caractérisation du gène responsable de la sclérose tubéreuse de Bourneville (TSC2) (m/s $\mathrm{n}^{\circ} 5$, vol. 10, p. 601) [1]), le Consortium Européen de recherche sur la polykystose rénale autosomique dominante de l'adulte (APRD1) vient d'isoler le gène responsable (PKD1) de cette maladie [2]. Les gènes TSC2 et PKD1 sont situés à proximité l'un de l'autre, sur le bras court du chromosome 16. L'APKD1 est l'une des maladies génétiques les plus fréquentes puisqu'elle atteint environ un individu sur 1000 (m/s n 9, vol.6, p. 904) La pathologie n'est pas limitée au rein; des kystes hépatiques, parfois pancréatiques, des anévrismes intracrâniens, des diverticuloses coliques et des valvulopathies cardiaques ont aussi été décrites à des fréquences variables. Cette affection est responsable en Europe comme aux USA de 8 à $10 \%$ des transplantations rénales. Le point de départ du clonage positionnel a été la localisation, il y a déjà neuf ans, du locus PKD1 sur le bras court du chromosome 16 (p13.3) en position centromérique par rapport au locus $\alpha$. globine [3]. Plus récemment un second locus (APKD2) a été localisé sur le bras long du chromosome 4 (q13-q23) [4]. On estime qu'environ $85 \%$ des formes d'APKD sont liées au locus PKD1.

Au cours de ces dernières années, la démarche a été classique; on a pu isoler de nouveaux marqueurs et délimiter ainsi le locus PKD1 dans une région d'environ $600 \mathrm{~kb}$, par des études familiales, et aussi grâce à l'utilisation d'hybrides somatiques. La densité des îlots CpG [5], et l'identification de nombreux transcrits de cette région indiquaient qu'il pouvait $y$ avoir au moins une vingtaine de gènes candidats pour l'APKD1 [6]. Des délétions associées au locus TSC2 avaient déjà permis d'indiquer la distribution génomique de certains d'entre eux et d'en isoler les transcrits. C'est principalement la duplication d'une partie de la région candidate qui a rendu difficile la découverte du gène PKD1.

L'observation capitale a été faite dans le groupe de P. Harris (IMM, Oxford, GB) qui a orienté récemment ses recherches vers l'étude submicroscopique des malades atteints d'APKD1. Cette équipe a mis en évidence une translocation équilibrée: $46 \times X t(16 ; 22)(p .13 .3 ; q 11.21)$ associée au phénotype APKD1 dans une famille portugaise. Les études d'hybridation in situ par marquage fluorescent et les cartographies par électrophorèse en champ pulsé ont localisé le point de cassure a environ $20 \mathrm{~kb}$ du gène TSC2.

Cette observation a permis de réduire considérablement le champ de recherche du meilleur gène candidat jusqu'à isoler enfin un transcrit anormal spécifique de la translocation. Le gène correspondant (préalablement noté 3A3) [1] devenait alors très fortement candidat. II a été analysé chez trois autres malades atteints de polykystose rénale. Deux délétions différentes et une anomalie de l'épissage ont été trouvées, démontrant ainsi qu'il s'agissait bien du gène PKD1. Bien que sa taille ne soit pas encore bien précisée, on s'attend à une étendue minimale de 40kb. Le gène PKD1 est transcrit en un ARN messager de $14 \mathrm{~kb}$ (PBP) dont il existe des homologues partiels liés à la duplication en 16p13.1 (l'hypothèse d'un épissage différentiel a été éliminée). Le gène PKD1 s'exprime non seulement dans le rein, mais aussi dans des lignées cellulaires de foie, de cerveau et dans les fibroblastes. La séquence protéique déduite de la séquence nucléotidique ne permet pas, pour l'instant, d'attribuer de fonction connue à la protéine PKD1.

L'analyse de plusieurs mutants du gène PKD1 et de délétions affectant à la fois TSC2 et PKD1 devraient nous aider a comprendre le mécanisme complexe des lésions de la polykystose rénale et apporter de nouvelles informations sur les "syndromes des gènes contigus" puisque TSC2 et PKD1 ne sont séparés que d'environ 2 kb!

11. The European Chromosome 16 Tuberous Sclerosis Consortium. Identification and characterization of the tuberous sclerosis gene on chromosome 16. Cell 1993; 75: 1305-15.J

12. The European Polycystic Kidney Disease Consortium. The polycystic kidney disease 1 gene encodes a $14 \mathrm{~kb}$ transcript and lies within a duplicated region on chromosome 16. Cell 1994 (sous presse).)

13. Reeders ST, Breuning MH, Davies KE, Nicholls RD, Jarman AP, Higgs DR, Pearson PL et al. A higly polymorphic DNA marker linked to adult polycystic kidney disease on chromosome 16. Nature $1985 ; 317: 542-4$.]

[4. Kimberling Wj, Kumar S, Gabow PA, Kenyon JB, Connolly CJ, Somlo S. Autosomal dominant polycystic kidney disease localisation of the second gene to chromosome 4q13-q2. Genomics 1993; 18:467-72.J

[5. Jordan B, llots HTF; le gène annoncé. médecine/sciences 1991; 7: 153-60.]

16. Germino GG, Weinstat-Saslow D. Hinunelbauer H, Gillepsie GA, Somio S, Wirth B, Barton N, Harris KN, Frischauf AM, Reeders ST. The gene for APKD1 lies in a $750 \mathrm{~kb}$ CpG rich region. Genomics 1992; 13: 144-51.J

\section{ERRATUM}

\section{Céruloplasmine et maladie de Wilson}

Une erreur de frappe s'est glissée dans le Tableau I de la mini-synthèse intitulée "La maladie de Wilson : le gène en cause est similaire au gène responsable de la maladie de Menkes" par Jamel Chelly, médecine/sciences $n^{\circ}$ 3, vol. 10, mars 1994. Dans la maladie de Wilson, comme dans la maladie de Menkes, la concentration de la céruloplasmine sérique est abaissée, et non pas augmentée comme l'indique le Tableau I. 